This is the "Authors Accepted Manuscript" of an article published in: Proceedings of the Combustion Institute, Volume 37, Issue 2, 2019, Pages 2493-2501; Available online 25 July 2018.

The final version of the article may be found at: https://doi.org/10.1016/j.proci.2018.07.031

\title{
Local entrainment velocity in a premixed turbulent annular jet flame
}

\author{
Luis Cifuentes ${ }^{\mathrm{a}, *}$, Andreas Kempf $^{\mathrm{a}, \mathrm{b}}$, Cesar Dopazo $^{\mathrm{c}}$ \\ ${ }^{a}$ Institute for Combustion and Gasdynamics (IVG), Chair of Fluid Dynamics, University of Duisburg-Essen, Carl-Benz Straße 199, 47057 \\ Duisburg, Germany \\ ${ }^{b}$ Center for Nano-Integration Duisburg-Essen (CENIDE), University of Duisburg-Essen, Carl-Benz Straße 199, 47057 Duisburg, Germany \\ ${ }^{c}$ School of Engineering and Architecture - Fluid Mechanics Area, University of Zaragoza, C/ Maria de Luna 3, 50018 Zaragoza, Spain
}

\begin{abstract}
The local entrainment velocity of the enstrophy interfaces of a methane-air turbulent premixed turbulent annular jet flame stabilized on a bluff-body burner has been investigated using a high-fidelity flame-resolved three-dimensional simulation. The enstrophy (inner and outer) and the scalar interfaces have been defined and characterized by their propagation speeds, $V_{E}$ and $S_{d}$, relative to the fluid flow. Mean values $\left(\left\langle S_{d} \mid c\right\rangle\right.$ and $\left.\left\langle V_{E} \mid c\right\rangle\right)$ conditioned on the reaction progress variable $c$ have been obtained. A thin layer (near the enstrophy interfaces) has been used to compute mean values ( $\left\langle V_{E} \mid E\right\rangle,\left\langle S_{d} \mid E\right\rangle$, and its different contributions) conditional upon enstrophy $E$. At the inner interface, results indicate that $\left\langle S_{d} \mid c\right\rangle>0$ and $\left\langle S_{d} \mid E\right\rangle>0$ (entrainment of fresh reactants into the flame front and hot products), while $\left\langle V_{E} \mid c\right\rangle<0$ and $\left\langle V_{E} \mid E\right\rangle<0$ (entrainment of hot products into the reacting jet across the inner enstrophy interface). The outer enstrophy interface displays $\left\langle V_{E} \mid E\right\rangle>0$ (ambient gases are predominantly entrained into the jet of reactants), which implies a lean mixture in its neighborhood. These preliminary results aim at understanding the physical mechanisms of flame anchoring, in terms of entrainments of either hot products or fresh reactants into the diffusive-reactive region. Local geometries of the inner and outer interfaces have also been examined, through the computation of joint probability density functions of the mean curvature $k_{m}$ and Gauss curvature $k_{g}$ of the isoenstrophy surfaces, and through $\left\langle V_{E} \mid k_{m}, k_{g}\right\rangle$ at the inner and outer interfaces. This information has subsequently been used to discuss the physics of how the turbulent entrainment process affects premixed flames.
\end{abstract}

Keywords: Turbulent entrainment process, turbulent/non-turbulent interface, iso-scalar non-material surfaces, enstrophy interface, turbulent premixed flame.

\footnotetext{
${ }^{*}$ Corresponding author:

Email address: luis.cifuentes@uni-due.de (Luis Cifuentes )
} 


\section{Introduction}

Turbulent/non-turbulent interfaces (T/NT) [1, 2] and thin flames [3] are examples of non-material isosurfaces across which transfer of fluid variables occur. Entrainment of fluid from the irrotational flow into the rotational turbulent region characterizes the T/NT interfaces, while fluxes of fresh gases and of thermal energy from the flame into the preheat zone typify premixed flames.

Corrsin and co-workers $[1,2]$ detected the T/NT interface in experiments and named it the 'laminar superlayer' (the thin region between irrotational and turbulent regions of the flow). They suggested that the superlayer thickness was of the order of the Kolmogorov micro-scale, $\eta$, and that viscous diffusion of vorticity towards the irrotational flow, enhanced by stretching of small eddies of sizes comparable to $\eta$, was responsible for the entrainment process. Libby [4] proposed an evolution equation, with an unknown source term, for the intermittency function, which takes the value one in the turbulent zone and zero in the irrotational region and displays a discontinuity at the T/NT interface. Dopazo and co-workers [5,6] related the intermittency source to the entrainment velocity and obtained consistent averaged transport equations for both the turbulent and non-turbulent zones, including interaction terms with a clear physical meaning. Dopazo [7] applied this intermittency formalism to turbulent diffusion flames.

The enstrophy $E=\left(\omega_{i} \omega_{i}\right) / 2$ (where $\omega_{i}$ stands for the vorticity vector) can be used as a scalar to define the T/NT interface. The enstrophy $E$ will be zero in the irrotational region and $E>0$ in the rotational turbulent zone. The enstrophy interface will be a thin layer where $0<E(\mathbf{x}, t) \leq \varepsilon$, where $\varepsilon$ is a small value of the enstrophy, whereas in the rotational region $E(\mathbf{x}, t)>\varepsilon$ (note the arbitrariness of this definition, dependent on the value of $\varepsilon$ ). The enstrophy interface will move in its normal direction, relative to the fluid, with a displacement speed, which causes irrotational fluid to progress across it with the entrainment velocity equal to the displacement speed and opposite sign. Large-scale eddy motions often fold separating interfaces between two fluid streams to engulf fluid from one stream into the other [8]. This 'ingested' fluid is 'digested' and incorporated into the second stream by molecular diffusion. A turbulent premixed flame front (scalar interface) moves with a displacement speed, relative to the fresh gases, entraining them into the preheat zone with a velocity equal to the displacement speed and opposite sign [3]. Hot fluid from the reaction zone is diffused into the preheat zone to warm up reactants as they move across the flame.

Notwithstanding the continuing experimental and numerical research on enstrophy interfaces [9-15], a comprehension of its physics is far from complete. Furthermore, the investigation of the entrainment process in variable-density turbulent reacting flows is limited in comparison to that of constant-density turbulence. Corrsin [1] observed that enstrophy and temperature interfaces are not coincident in a turbulent heated jet. A joint examination of T/NT and scalar interfaces in turbulent premixed flames might shed some light on various processes in which strong interactions between small-scale vortical structures and scalar topologies will be relevant. The stabilization mechanism of a lean methane-air premixed turbulent annular jet flame on a bluff-body burner [16-18] might be a good example; the interaction between regions of hot recirculating gases and of fresh reactants, responsible for anchoring the flame to the burner, involves entrainments across scalar and enstrophy interfaces, which are worth investigating.

This work aims at examining scalar and enstrophy interfaces in a turbulent premixed flame stabilized behind a bluff-body and comparing the flame displacement speed $S_{d}$ and the local entrainment velocity $V_{E}$. This study also explores the various contributions to both $V_{E}$ and $S_{d}$. The effects of the dilatation rate, the viscous torque and the spatial variation of viscosity on $V_{E}$ have never been taken into account in previous studies and are studied in this paper. The present investigation sheds some light on the nature of flame stabilization because the analysis $i$ ) gives information about the engulfment and digestion by molecular diffusion of hot products and fresh gases near the burner exit, ii) can provide details on how the local equivalence ratio of the fresh mixture affects the flame anchoring, and iii) presents new ideas to describe and quantify transport processes across the inner enstrophy and scalar interfaces that influence the flame stabilization. Furthermore, in this study the local entrainment velocity is related to the shape of the enstrophy interface to examine the dependence of the enstrophy transport on its curvature. To the best of the authors knowledge, a detailed joint study of the scalar field and local enstrophy geometries for variable-density turbulent reacting flows has never been reported.

\section{Flame resolved simulation database}

A detailed flame resolved simulation database [19, 20] of the Cambridge setup of a lean methane-air premixed turbulent annular jet flame stabilized behind a bluff-body burner is examined. This flame has 
been investigated by Barlow, Hochgreb and their coworkers [16-18] experimentally and numerically via Large Eddy Simulations (LES) [21-23]. The burner supplies two co-annular streams of methane-air mixtures at ambient conditions surrounding the central bluff-body. The two streams are embedded in a coflow of air with a velocity of $0.4 \mathrm{~m} / \mathrm{s}$. The flame resolved simulation database examined here was generated by Proch $[19,20]$ using the PsiPhi code on a grid of 1.6 billion cells with a grid resolution of $100 \mu \mathrm{m}$ throughout the domain. The Lewis number was unity for all species and the chemistry was tabulated with the Premixed Flamelet Generated Manifolds (PFGM) approach [24, 25]. Interested readers are referred to Proch's work [19, 20] for detailed explanations of the numerical implementation of this simulation. Table 1 gives the inlet flow velocities of the co-annular streams $u$, the integral length scale $l$, root-mean-square turbulent velocity fluctuation $u^{\prime}$, the equivalence ratio $\phi=$ 0.75, Damköhler number $D a=l S_{L} / u^{\prime} \delta_{L}$, the laminar flame speed $S_{L}$, the unstretched laminar flame thickness $\delta_{L}$ and the turbulent Reynolds number $R e_{T}=u^{\prime} l / v$, where $v$ is the kinematic viscosity that depends on the temperature. The unstretched laminar flame thickness $\delta_{L}=\left(T_{b}-T_{u}\right) / \max |\nabla T|_{L}$ is based on the maximum temperature gradient and the temperatures in the unburned, $T_{u}$, and burned, $T_{b}$, gas. The Kolmogorov length is $\eta=41 \mu \mathrm{m}$ at the inlet and increases significantly downstream and from the unburned to the burned side of the flame. The ratio of the grid resolution to the Kolmogorov micro-scale in the reaction zone is $\Delta / \eta \approx 1$. The thickness of the enstrophy interface is of the order of the Taylor scale $[11,12,26]$, which means that the thickness of the enstrophy interface is well resolved for the present investigation. Results are analyzed at $t=0.34 \mathrm{~s}$, which is much greater than one initial integral eddy turnover time $\tau_{l}=60 \times 10^{-5} \mathrm{~s}$ (based on the inner stream rms of velocity fluctuations) and more than one flow-through time $t=0.28 \mathrm{~s}$ (based on the air coflow velocity). As shown by Proch et al. [19, 20], combustion takes place in the thin reaction zones regime [3] on the side of the fresh gases and moves towards the corrugated flamelet regime on the burned side.

\begin{tabular}{ccccccccccc}
\multicolumn{1}{c}{ Table 1: Parameters of the inner and outer reactants streams. } \\
\hline Stream & $\begin{array}{c}u \\
\mathrm{~m} / \mathrm{s}\end{array}$ & $\begin{array}{c}u^{\prime} \\
\mathrm{mm}\end{array}$ & $\begin{array}{c}S_{L} \\
\mathrm{~m} / \mathrm{s}\end{array}$ & $\begin{array}{c}\delta_{L} \\
\mathrm{~m} / \mathrm{s}\end{array}$ & $\begin{array}{c}\eta \\
\mathrm{mm}\end{array}$ & $\mu \mathrm{m}$ & - & - & - & - \\
\hline Inner & 8.31 & 0.5 & 0.9 & 0.21 & 0.56 & 41 & 0.75 & 0.2 & 28.1 \\
\hline Outer & 18.7 & 0.5 & 1.8 & 0.21 & 0.56 & 25 & 0.75 & 0.1 & 56.3 \\
\hline
\end{tabular}

\section{Enstrophy and scalar interfaces}

The interfaces considered in this work are nonmaterial surfaces, which propagate relative to the fluid with spatially and temporally varying speeds. Enstrophy $E(\mathbf{x}, t)$ and reaction progress variable $c(\mathbf{x}, t)=\left(Y_{c}-\right.$ $\left.Y_{c}^{\min }\right) /\left(Y_{c}^{\max }-Y_{c}^{\min }\right)$ are used to characterize the interfaces of interest $\left(Y_{c}=Y_{\mathrm{CO} 2}+Y_{\mathrm{C}}+Y_{\mathrm{H} 2 \mathrm{O}}\right.$, [19, 20]). The progress variable $c(\mathbf{x}, t)$ is zero in the fresh reactants and unity in the fully burnt products. An isosurface $c(\mathbf{x}, t)=\Gamma^{+} \rightarrow 0$ will define the interface, that instantaneously separates fresh gases from the reacting region. An enstrophy or T/NT interface can be identified as the mathematical iso-surface $E(\mathbf{x}, t)=\varepsilon$, where $\varepsilon$ is a given enstrophy infinitesimal value [10-13, 27]; this interface approximately separates rotational turbulent regions from the irrotational non-turbulent flow.

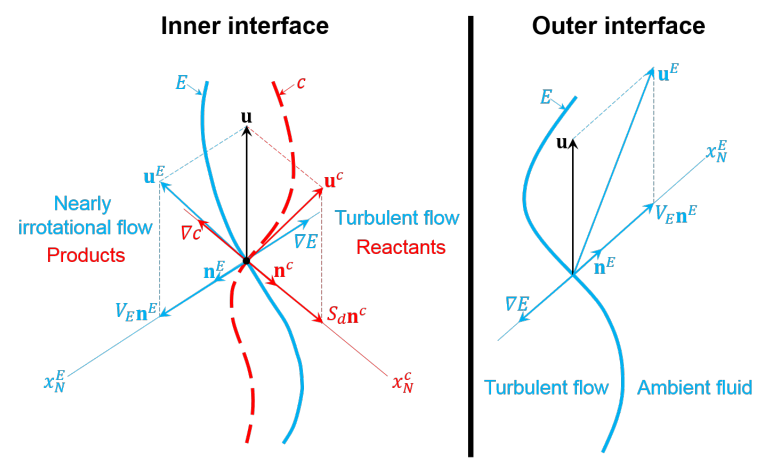

Figure 1: Schematic representation of non-material iso-scalar and isoenstrophy surfaces at the inner and outer interfaces.

The absolute velocities of the enstrophy and scalar interfaces are expressed as $\mathbf{u}^{E}=\mathbf{u}+V_{E} \mathbf{n}^{E}$ and $\mathbf{u}^{c}=$ $\mathbf{u}+S_{d} \mathbf{n}^{c}$, respectively. Here, $\mathbf{u}$ is the local fluid velocity, $\mathbf{n}^{E}=-\nabla E /|\nabla E|=\left(\partial E / \partial x_{i}\right) /\left(\partial E / \partial x_{N}^{E}\right)$ and $\mathbf{n}^{c}=-\nabla c /|\nabla c|=\left(\partial E / \partial x_{i}\right) /\left(\partial c / \partial x_{N}^{c}\right)$ are unit vectors normal to $E(\mathbf{x}, t)=\varepsilon$ and $c(\mathbf{x}, t)=\Gamma$, where $x_{N}^{E}$ and $x_{N}^{c}$ are the coordinates in the normal direction to the iso-enstrophy surface and the iso-scalar surface, respectively. The magnitudes $V_{E}$ and $S_{d}$ are the propagation velocities of the enstrophy and scalar interfaces relative to the flow velocity (see Fig. 1). An enstrophy interface moves on the average towards the irrotational flow, causing the growth of the turbulent region. The entrainment velocity is then $-V_{E} \mathbf{n}^{E}$ and $V_{E} \mathrm{~S}$ is the flow rate of entrained fluid from the irrotational zone across a surface element $\mathrm{S}$. On the other hand $S_{d} \mathbf{n}^{c}$ is the displacement velocity of the scalar interface towards the fresh gases and $-S_{d} \mathbf{n}^{c}$ is the entrainment velocity of fresh reactants towards the flame region. The propagation speeds $V_{E}$ and $S_{d}$ can be expressed as [10, 27-34]: 


$$
\begin{aligned}
& V_{E}(\mathbf{x}, t)=\underbrace{\frac{\omega_{i} S_{i j} \omega_{j}}{|\nabla E|}}_{V_{E 1}} \underbrace{-\frac{2 E S_{j j}}{|\nabla E|}}_{V_{E 2}} \underbrace{+\frac{\varepsilon_{i j k} \frac{\omega_{i}}{\rho^{2}} \frac{\partial \rho}{\partial x_{j}} \frac{\partial p}{\partial x_{k}}}{|\nabla E|}}_{V_{E 3}} \\
& \underbrace{-\frac{\varepsilon_{i j k} \frac{\omega_{i}}{\rho^{2}} \frac{\partial \rho}{\partial x_{j}} \frac{\partial \tau_{k l}}{\partial x_{l}}}{|\nabla E|}}_{V_{E 4}}+\underbrace{+\frac{\nu\left(\nabla^{2} E\right)}{|\nabla E|}}_{V_{E 5}}-\underbrace{-\frac{v\left(\frac{\partial \omega_{i}}{\partial x_{j}} \frac{\partial \omega_{i}}{\partial x_{j}}\right)}{|\nabla E|}}_{V_{E 6}} \\
& +\frac{1}{|\nabla E|}\left\{\frac { 1 } { \rho } \frac { \partial \mu } { \partial x _ { j } } \left[\frac{\partial E}{\partial x_{j}}+\varepsilon_{i j k} \omega_{i}\left(\nabla^{2} u_{k}\right)\right.\right. \\
& \underbrace{\left.\left.+\varepsilon_{i j k} \omega_{i} \frac{\partial\left(S_{l l}\right)}{\partial x_{k}}\right]+\frac{2}{\rho} \frac{\partial^{2} \mu}{\partial x_{j} \partial x_{l}} \varepsilon_{i j k} \omega_{i} S_{l k}\right\}}_{V_{E 7}}
\end{aligned}
$$

$$
S_{d}(\mathbf{x}, t)=\underbrace{\frac{1}{|\nabla c|} \frac{1}{\rho} \frac{\partial}{\partial x_{j}}\left(\rho D \frac{\partial c}{\partial x_{j}}\right)}_{V_{c 1}}+\underbrace{\frac{1}{|\nabla c|} \frac{\dot{\omega}}{\rho}}_{V_{c 2}}
$$

In Eq. (1), $S_{i j}$ is the strain rate tensor, $\varepsilon_{i j k}$ is the LeviCivitta alternating tensor, $u_{i}$ is the flow velocity vector, $\rho$ and $p$ stand for fluid density and pressure, $\tau_{i j}$ is the viscous stress tensor, $\mu$ is the dynamic viscosity, and $v$ is the kinematic viscosity. The term $V_{E 1}$ is the contribution to $V_{E}$ of the vortex stretching generation and $V_{E 2}$ is the propagation by enstrophy annihilation/production due to the volumetric dilatation rate $\nabla \cdot \mathbf{u}=S_{j j}$. Terms $V_{E 3}$ and $V_{E 4}$ represent the contributions to $V_{E}$ of the torques of pressure (baroclinic) and viscous forces, respectively. The term $V_{E 5}$ gives the displacement speed by viscous transport of enstrophy and $V_{E 6}$ is that due to the viscous dissipation of $E$. The term $V_{E 7}$ represents the contribution to $V_{E}$ of spatial variations of the dynamic viscosity. In Eq. (2), $D$ is the diffusivity coefficient of the progress variable and $\dot{\omega}$ its chemical source term. The terms $V_{c 1}$ and $V_{c 2}$ are the contributions to $S_{d}$ of the molecular diffusion rate and the chemical conversion of $c$, respectively. Results in this paper are presented in a dimensionless form such that: $E^{*}(\mathbf{x}, t)=E / E_{\max }$, $S_{d}^{*}=S_{d} / S_{L}, V_{E}^{*}=V_{E} / S_{L}$, and $V_{E \alpha}^{*}=V_{E \alpha} / S_{L}$ (with $\alpha=1,2 \ldots 7$ ), and $V_{c \alpha}^{*}=V_{c \alpha} / S_{L}$ (with $\left.\alpha=1,2\right)$, where $S_{L}=0.21 \mathrm{~m} / \mathrm{s}$.

\section{Local geometry of iso-enstrophy and iso-scalar surfaces}

The local iso-enstrophy and iso-scalar geometries can be characterized in terms of their mean curvature $k_{m}$ and

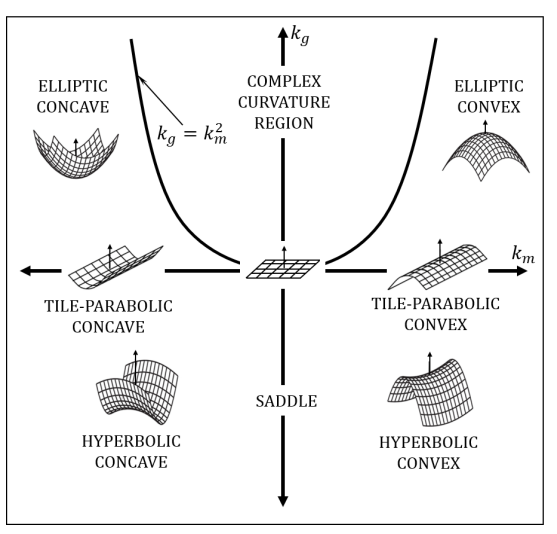

Figure 2: Classification of iso-surface geometries in terms of their mean curvature $k_{m}$ and Gauss curvature $k_{g}$ [35-37].

Gauss curvature $k_{g}$ [35]. Iso-surface curvature plays an important role in the enstrophy and in the scalar conservation equations since it can appear explicitly in the terms of tangential viscous and diffusive transports. Hence, it is important to study these topologies and quantify their effects on the enstrophy and scalar interfaces. Figure 2 [35-37] is included in this paper to facilitate the discussion related to the iso-surface geometries in the $k_{m}-k_{g}$ plane [35-37]. The region $k_{g}>k_{m}^{2}$ implies non-physical (complex) curvatures. Results in this paper are presented in a dimensionless form for $k_{m}^{*}=k_{m} \eta$ and $k_{g}^{*}=k_{g} \eta^{2}$, where $\eta=41 \mu \mathrm{m}$.

\section{Results and discussion}

Figure 3 shows an instantaneous enstrophy field, the enstrophy inner interface $\left(E^{*}=7.0 \times 10^{-5}\right)$ and outer interface $\left(E^{*}=1.0 \times 10^{-7}\right)$ and the progress variable isolines $c=0.1$ and $c=0.9$ (different enstrophy thresholds were tested to determine the enstrophy values that reliably detect the enstrophy interfaces). Figure 3 indicates that the flame-turbulence interaction is apparently weak near the burner exit, with the flame propagating in a low turbulence environment. Vortex shedding, at the edges of the bluff body and of the inner and outer walls of the tubes guiding the co-annular jets, generates a complicated flow pattern, but needs a certain axial distance to induce intense turbulence and strong wrinkling of the enstrophy and scalar interfaces. Interactions become stronger downstream, where the flame burns within the turbulent shear layer between hot products and the inner annular jet. The flame may contribute to increase enstrophy levels. It is worth noting that the outer enstrophy interface is more convoluted than the inner one, due to low temperatures, which reduce the turbulent kinetic 
energy dissipation rate. Figure 4 presents instantaneous radial profiles of $E^{*}$ and $c$. It can be noticed that in the hot products region, where $c=1$, the enstrophy levels are two to three orders of magnitude smaller than those in the region of high vortical activity, as the flame front causes a quasi-laminarization of the flow.

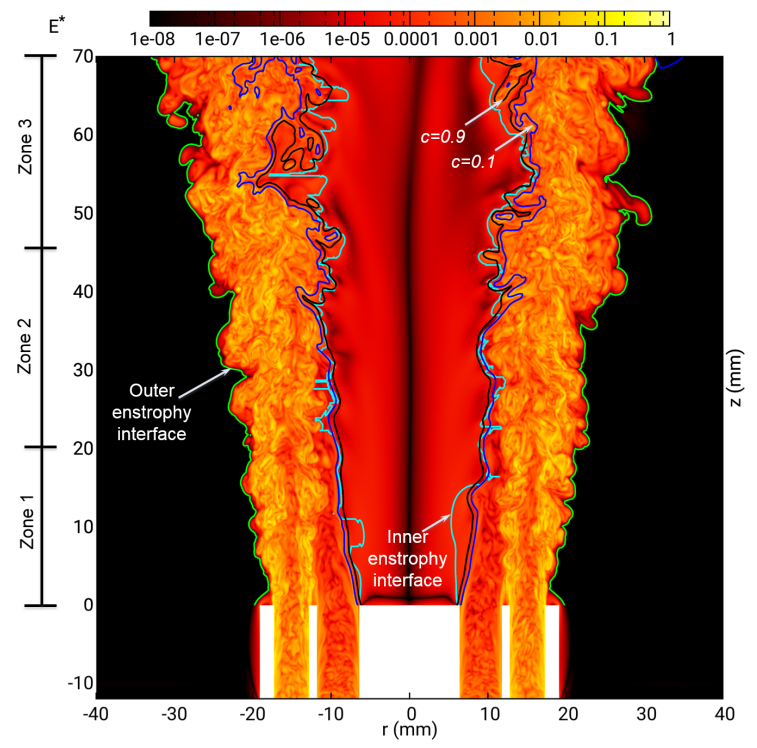

Figure 3: Instantaneous enstrophy field, progress variable iso-lines $c=0.1$ and $c=0.9$, and iso-lines defining the enstrophy inner $E^{*}=$ $7.0 \times 10^{-5}$ and outer $E^{*}=1.0 \times 10^{-7}$ interfaces.

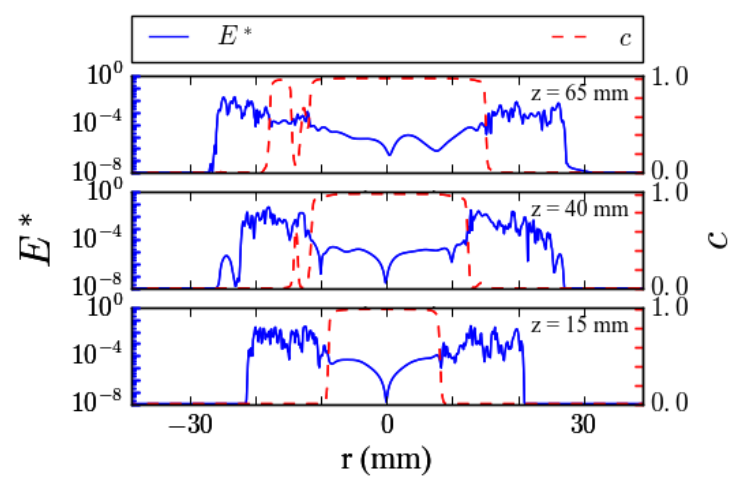

Figure 4: Instantaneous radial profiles of $E^{*}$ and $c$ at three different axial distances from the burner exit.

Further, the flame brush is defined as the region $0.01 \leq c \leq 0.99$. To evaluate differences in scalarenstrophy interactions, results are separately examined in three zones. Zone 1 extends over $0 \mathrm{~mm}<z \leq 20 \mathrm{~mm}$, Zone 2 over $20 \mathrm{~mm}<z \leq 45 \mathrm{~mm}$ and Zone 3 over $45 \mathrm{~mm}<z \leq 70 \mathrm{~mm}$, as illustrated in Figure 3. Data were analyzed for ten cross-stream planes $r-z$ through the burner midsection, considering grid points in a region $\pm 5 \mathrm{~mm}$ in the radial direction from the enstrophy inner and outer interfaces. The sensitivity of results to the thickness of that 'layer' $( \pm 5 \mathrm{~mm})$ is small; thicknesses of \pm 2 and $\pm 4 \mathrm{~mm}$ yield similar results to those presented here.

\subsection{Analysis of the local entrainment velocity and dis- placement speed across the scalar and enstrophy interfaces}

The normalized flame displacement speed $S_{d}^{*}$, entrainment velocity $V_{E}^{*}$ and the various contributions to $S_{d}^{*}$ and $V_{E}^{*}$ are plotted against the progress variable and enstrophy in Figure 5. Variables conditional upon $c$ are only shown for the inner enstrophy interface, as there is no flame near the outer one. Ensemble averaging is performed on a given iso-surface at the time $t=0.34 \mathrm{~s}$ when the statistics were extracted, the same approach has been adopted in several previous analyses [3]. It is apparent that the term $V_{c 1}^{*}$, the molecular diffusion contribution to $S_{d}^{*}$, is small towards the fresh gases and negative towards the hot products. The chemical reaction term $V_{c 2}^{*}$ is always positive and reaches its highest magnitudes for values of $c$ with high chemical conversion [19], which is in agreement with other threedimensional DNS results $[32,38]$ of turbulent premixed flames. It is worth stressing that the constant value of $S_{L}$ determined from the laminar flame computation only includes the effects of the chemical reaction rate. Therefore, a priori, $V_{c 2}^{*}=V_{c 2} / S_{L}$ should be of order one (it should be noticed that $V_{c 1}^{*}$ and $V_{c 2}^{*}$ have been scaled by 3 in Figure 5 and reach a peak of the order of unity). However, $S_{d}^{*}=V_{c 1}^{*}+V_{c 2}^{*}$ displays a maximum of about 0.23 for $c \approx 0.88$ in Zone 3. The flame displacement speed $S_{d}^{*}$ slightly increases in the downstream direction, and monotonically intensifies towards the burnt gases, as the iso-scalar surfaces located in the burning region propagate faster than those in the fresh gases. Consequently, two iso-scalar surfaces $c(\mathbf{x}, t)=\Gamma$ and $c(\mathbf{x}+\mathbf{r}, t)=\Gamma+\Delta \Gamma$ (where $\left.\mathbf{r}=\Delta x_{N} \mathbf{n}^{c}\right)$, for example in the preheat region, move towards the reactants and tend to reduce their distance $\left(\Delta x_{N}\right)$, which enhances the scalar-gradients [32, 38], apart from flow strain rates. Local entrainment velocities and displacement speeds are of the same order of magnitude across the flame, although $V_{E}^{*}$ is negative across the flame brush and increases its magnitude towards the burnt gases. Since $\mathbf{n}^{E}$ points towards the hot products, the high negative values of $V_{E}^{*}$ indicate that the inner enstrophy interface moves towards fresh gases, which means that fresh gases, with 


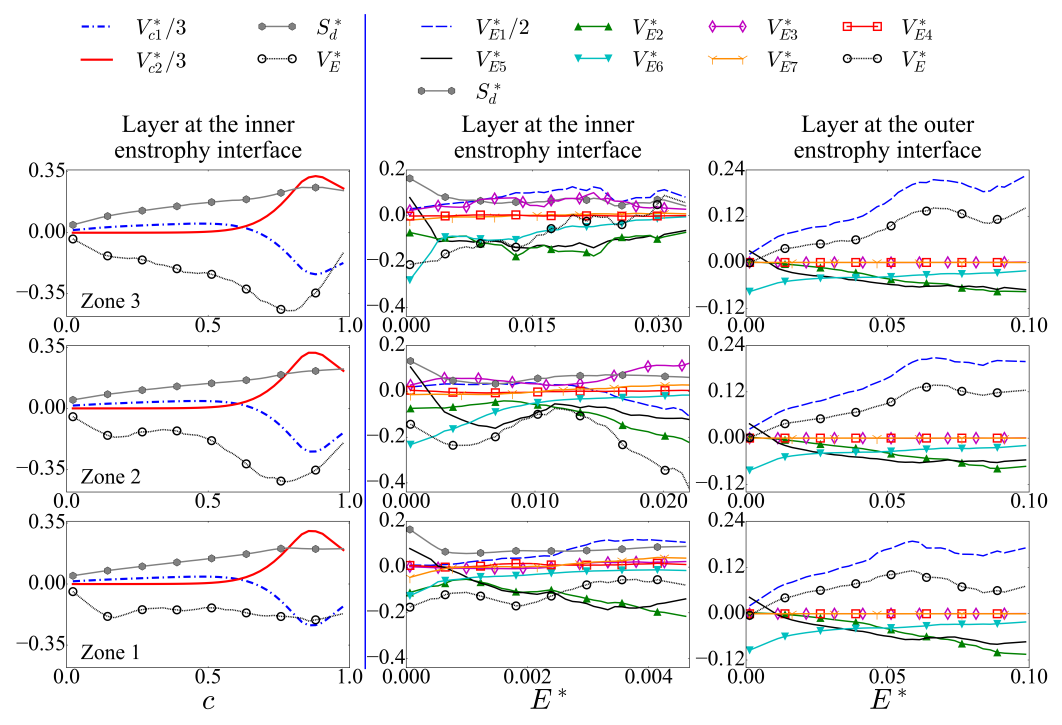

Figure 5: Mean values of the displacement speed $S_{d}^{*}$, the entrainment velocity $V_{E}^{*}$ and the different contributions to $S_{d}^{*}$ and $V_{E}^{*}$, conditional upon $c$ and $E^{*}$ (the mean values of $V_{c 1}^{*}$ and $V_{c 2}^{*}$ have been scaled by three, whereas $V_{E 1}^{*}$ has been divided by two).

enstrophies above the inner enstrophy threshold, are entrained into the hot product zone across the inner enstrophy interface; this implies that the vortical structures in the reacting jet region significantly reduce their intensities as they cross the inner enstrophy interface toward the recirculation zone of hot products. Analogously, positive values of $S_{d}^{*}$ also denote entrainment of reactants into the hot products across the scalar interface; these reactants rapidly disappear as they cross the scalar interface. These differences between $S_{d}^{*}$ and $V_{E}^{*}$ are consistent with the initial observation of Corrsin [1] of different enstrophy and scalar interfaces.

Figure 5 also shows conditional means upon the enstrophy for the layers about the inner and outer enstrophy interfaces. The samples obtained for the layer at the inner enstrophy interface range from $E^{*}=0$ to 0.004 in Zone 1, from $E^{*}=0$ to 0.02 in Zone 2 and $E^{*}=0$ to 0.03 in Zone 3. Near the outer interface, the range is $0.0-0.1$ throughout all zones. It is apparent that the ranges of enstrophy analyzed for the layer at the inner enstrophy interface increase from Zone 1 to Zone 3, with therefore more wrinkling and folding of the scalar and enstrophy interfaces and intense engulfment and entrainment. The displacement speed remains approximately constant for all values of $E^{*}$ except near zero enstrophy, $V_{E}^{*}$ is negative and increases from the irrotational flow to the rotational region in Zone 1 and 3 , but increases to a relative maximum of $V_{E}^{*} \approx 0.08$ at $E^{*} \approx 0.13$, decreasing for large values of enstrophy.
Figure 5 shows that the contribution to $V_{E}^{*}$ of the vortexstretching, $V_{E 1}^{*}=\{[|\boldsymbol{\omega}||\boldsymbol{S} \cdot \boldsymbol{\omega}| \cos (\boldsymbol{\omega},(\boldsymbol{S} \cdot \boldsymbol{\omega}))] /|\nabla E|\} / S_{L}$, is the leading positive term for all values of $E^{*}$ in Zone 1 and 3, but becomes negative for $E^{*}>0.18$ in Zone 2. $V_{E 1}^{*}$ depends on the magnitudes of the two vectors, vorticity $|\omega|$ and vortex-stretching $|\boldsymbol{S} \cdot \boldsymbol{\omega}|$, and on the cosine of the angle between them. Results for these quantities at both enstrophy interfaces are not explicitly shown here, but the alignment between $\boldsymbol{\omega}$ and $\boldsymbol{S} \cdot \boldsymbol{\omega}$ lessens from the turbulent jet region to the irrotational flow of recirculating products, and the magnitudes of $|\omega|$ and $|\boldsymbol{S} \cdot \boldsymbol{\omega}|$ increase towards the turbulent fluid, especially the vortex-stretching magnitude $|\boldsymbol{S} \cdot \boldsymbol{\omega}|$ in the shear layer between the inner and outer streams. Enstrophy is annihilated by the volumetric dilatation term, $V_{E 2}^{*}=-\left[\left(2 E S_{j j}\right) /|\nabla E|\right] / S_{L}$, at both enstrophy interfaces. High negative values of $V_{E 2}^{*}$ at the enstrophy interface are a consequence of the flame heat release, with $S_{j j}>0$, whereas a smaller contribution is apparent at the outer interface far from the flame. Contribution to $V_{E}^{*}$ of torques of pressure and viscous forces, $V_{E 3}^{*}$ and $V_{E 4}^{*}$, are negligible near the outer enstrophy interface with very small positive values at the inner interface; wrinkling of the inner interface increases downstream, induces a misalignment of $\nabla \rho$ and $\nabla p$ and increases the baroclinic torque, in agreement with previous numerical results $[34,39]$. The viscous transport of enstrophy, $V_{E 5}^{*}=\left\{\left[v\left(\nabla^{2} E\right)\right] /|\nabla E|\right\} / S_{L}$, is positive for small enstrophy values near the irrotational zone, but nega- 
tive over most values of the chosen $E^{*}$ ranges about the inner and outer enstrophy thresholds; $E^{*}\left(x_{N}\right) \leq 1$ increases monotonically towards the jet of reactants and $\nabla^{2} E>0$ below $E^{*}=7.0 \times 10^{-5}$, with an inflection point $\left(\nabla^{2} E=0\right)$ of the $E^{*}\left(x_{N}\right)$ profile where $V_{E 5}^{*}=0$. The term $V_{E 5}^{*}$ is negative above $E^{*}=7.0 \times 10^{-5}$, where $\nabla^{2} E<0$. The contribution to $V_{E}^{*}$ of viscous dissipation, $V_{E 6}^{*}=-\left\{\left[v\left(\partial \omega_{i} / \partial x_{j}\right)\left(\partial \omega_{i} / \partial x_{j}\right)\right] /|\nabla E|\right\} / S_{L}$, is negative and its magnitude decreases from the region in the neighborhood of $E^{*}=7.0 \times 10^{-5}$ (where high values of $|\nabla E|$ occur) as $E^{*}$ increases. The term $V_{E 7}^{*}$ due to spatial variations of the dynamic viscosity is either small or negligible for all values of $E^{*}$.

\subsection{Entrainment velocity conditioned on the local ge- ometry of the enstrophy interfaces}

It is interesting to scrutinize the dependence of the entrainment velocity $V_{E}^{*}$ on the local shape of the enstrophy interfaces. Figure 6 shows the joint probability density functions (JPDFs) of the mean curvature $k_{m}^{* E}$ and Gauss curvature $k_{g}^{* E}$ of the enstrophy interfaces (left side of Figure 6) for various regions of the computational domain. Nearly planar enstrophy interfaces are most probable (see Figure 2 for the classification), in agreement with experimental results [10]. JPDFs with longer tails occur at the outer interface, whereas the proximity of the flame reduces the maximum values of both curvatures. It is worth stressing that the wrinkling length scale (radius $=1 / k_{m}$ ) of the smallest geometry is greater than the Kolmogorov length micro-scale, which means that the curvatures of the iso-enstrophy surfaces are well resolved. Statistical distributions are slightly skewed towards $k_{m}^{* E}>0$, which indicates a higher probability of finding convex micro-structures, especially of an elliptic nature (convex cups). Curvature values of geometries with parabolic cylinder features seem not to vary in the different zones, whereas the probability of elliptic concave geometries decreases downstream. The long tails of the outer interface JPDFs indicate a strong entrainment across the interface, with engulfment and subsequent digestion by molecular diffusion of irrotational fluid pockets of ambient fluid into the fresh gases; this changes the local equivalence ratio and keeps a very lean mixture near the outer enstrophy interface.

Figure 6 also depicts the entrainment velocity $V_{E}^{*}$ conditional upon $k_{m}^{* E}$ and $k_{g}^{* E}$. Values of $V_{E}^{*}>0$ (entrainment of hot products into reactants) close to the inner enstrophy interface are associated with elliptic-concave geometries with large magnitudes of $k_{m}^{* E}$, whereas slightly concave, nearly flat and convex structures display mainly $V_{E}^{*}<0$ (entrainment of fresh reactants into hot products). On the other hand, in the neighborhood of the outer enstrophy interface, $V_{E^{*}}>0$ (entrainment of ambient fluid into the jet of reactants) correlates with elliptic convex micro-structures, whereas $V_{E}^{*}<0$ (detrainment of reactants into the ambient co-flow) is associated with concave geometries. This enhances the convolution of the outer enstrophy interface since microstructures with different shape propagate in opposite direction increasing the surface area of the outer enstrophy interface.

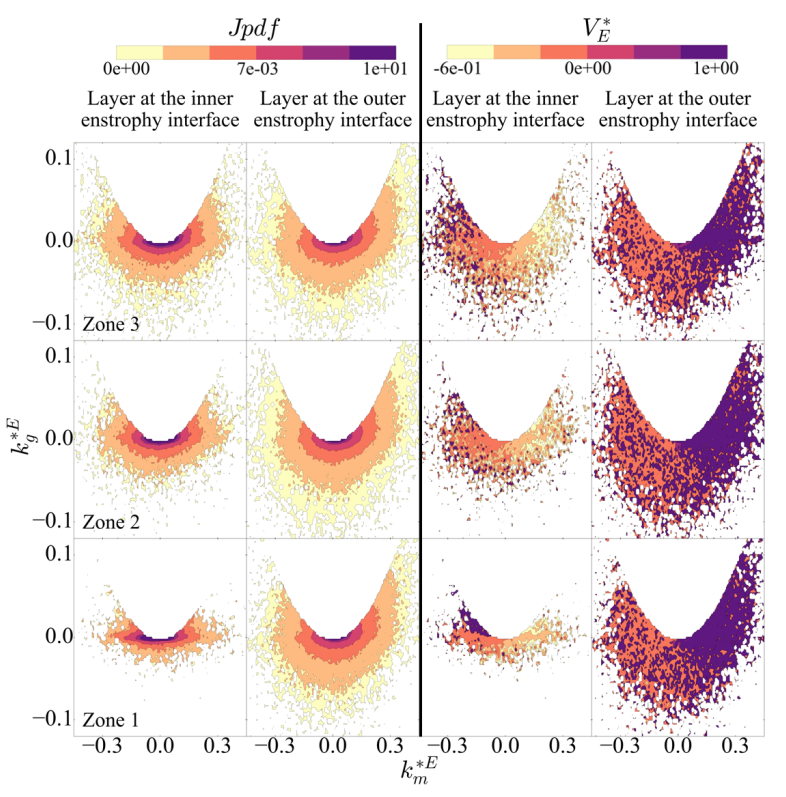

Figure 6: JPDFs of $k_{m}^{* E}$ and $k_{g}^{* E}$ (left side) of the iso-enstrophy surfaces, and the entrainment velocity $V_{E}^{*}$ conditional upon $k_{m}^{* E}$ and $k_{g}^{* E}$ (right side).

\section{Conclusions}

The enstrophy (inner and outer) and the scalar interfaces have been defined and characterized by their propagation speeds, $V_{E}$ and $S_{d}$, relative to the fluid flow. Mean values of $S_{d}$ and $V_{E}$ conditioned on the reaction progress variable $c$ and enstrophy $E$ in the neighborhood of the enstrophy interfaces have been obtained. The local entrainment velocity has been correlated with smallscale geometrical features of the enstrophy interfaces.

At the inner enstrophy interface, the mean conditional values are $\left\langle S_{d} \mid c\right\rangle>0$ (entrainment of fresh reactants into the flame front) and $\left\langle V_{E} \mid c\right\rangle<0$ (entrainment of hot products into the flame front). On the other hand, $\left\langle S_{d} \mid E\right\rangle>0$ (entrainment of fresh mixture into hot products across the inner enstrophy interface) and $\left\langle V_{E} \mid E\right\rangle<0$ (entrainment of hot products into the jet 
of reactants); vortical structures have a negligible intensity in the hot products, where $\left\langle V_{E} \mid E\right\rangle<0$ for small values of enstrophy. The outer enstrophy interface displays $\left\langle V_{E} \mid E\right\rangle>0$, which indicates that ambient gases are predominantly entrained into the jet of reactants. At the inner enstrophy interface, it was found that positive values of $\left\langle V_{E} \mid k_{m}, k_{g}\right\rangle$ (entrainment of hot products into the jet of reactants) are associated to elliptic-concave enstrophy micro-structures, whereas $\left\langle V_{E} \mid k_{m}, k_{g}\right\rangle<0$ (entrainment of unburnt gases into hot products) correlates with slightly concave, flat and convex geometries. At the outer enstrophy interface, maximum values of $\left\langle V_{E} \mid k_{m}, k_{g}\right\rangle>0$ (entrainment of ambient gases into the reacting jet) correlate with convex enstrophy microstructures, whereas concave geometries are associated with small values of $\left\langle V_{E} \mid k_{m}, k_{g}\right\rangle<0$ (entrainment of unburnt gases into the ambient fluid). The outer interface predominantly propagates towards the ambient fluid $\left(\left\langle V_{E} \mid E\right\rangle>0\right.$ and $\left.\left\langle V_{E} \mid k_{m}, k_{g}\right\rangle>0\right)$, which means that local entrainment of ambient air into the turbulent fresh mixture reduces the local equivalence ratio and creates a lean mixture near that interface.

Although the scalar and enstrophy interfaces are different [1], entrainment processes or mixing of fresh reactants and hot products are characterized by $S_{d}$ and $V_{E}$. These preliminary results can help to understand how turbulent entrainment processes affect the physical mechanisms of flame anchoring near the burner exit. Modifying the correct balance of entrainment of hot products and fresh reactants, for instance, by increasing mean flow velocity and turbulence intensity, can lead to flame liftoff or blowout. Future investigation will address the influence of entrainment processes on chemical reaction rates in various zones of the flame. A better comprehension of these physical mechanisms should better guide the formulation of sound and accurate mixing and combustion models.

\section{Acknowledgments}

The authors are grateful to Jülich Supercomputing Centre (JSC) for computational support. This project has received funding from the European Union's Horizon 2020 research and innovation program under grant agreement No 706672 - ITPF.

\section{References}

[1] S. Corrsin, NACA Report No W-94 (1943).

[2] S. Corrsin, A. Kistler, NACA Report No 12441244 (1954).

[3] T. Poinsot, D. Veynante, Theoretical and numerical combustion, RT Edwards, 2005.
[4] P. A. Libby, J. Fluid Mech. 68 (1975) 273-295.

[5] C. Dopazo, J. Fluid Mech. 81 (1977) 433-438.

[6] E. E. O'Brien, C. Dopazo, Structure and Mechanisms of Turbulence II (1978) 124-137.

[7] C. Dopazo, Acta Astron. 6, 999-1004 (1977).

[8] G. L. Brown, A. Roshko, J. Fluid Mech. 64 (1974) 775-816.

[9] J. P. Mellado, L. Wang, N. Peters, J. Fluid Mech. 626 (2009) 333-365.

[10] M. Wolf, B. Lüthi, M. Holzner, D. Krug, W. Kinzelbach, A. Tsinober, Phys. Fluids 24 (2012) 105110.

[11] D. K. Bisset, J. C. Hunt, M. M. Rogers, J. Fluid Mech. 451 (2002) 383-410.

[12] J. Westerweel, C. Fukushima, J. Pedersen, J. Hunt, Phys. Rev. Lett. 95 (2005) 174501.

[13] M. Holzner, A. Liberzon, N. Nikitin, W. Kinzelbach, A. Tsinober, Phys. Fluids 19 (2007) 071702.

[14] C. B. da Silva, J. C. Pereira, Phys. Fluids 20 (2008) 055101.

[15] F. Hunger, M. Gauding, C. Hasse, J. Fluid Mech. 802 (2016).

[16] M. S. Sweeney, S. Hochgreb, M. J. Dunn, R. S. Barlow, Combust. Flame 159 (2012) 2896-2911.

[17] M. S. Sweeney, S. Hochgreb, M. J. Dunn, R. S. Barlow, Combust. Flame 159 (2012) 2912-2929.

[18] M. M. Kamal, R. S. Barlow, S. Hochgreb, Combust. Flame 162 (2015) 3896-3913.

[19] F. Proch, P. Domingo, L. Vervisch, A. M. Kempf, Combust. Flame 180 (2017) 321-339.

[20] F. Proch, P. Domingo, L. Vervisch, A. M. Kempf, Combust. Flame 180 (2017) 340-350

[21] S. Nambully, P. Domingo, V. Moureau, L. Vervisch, Combust. Flame 161 (2014) 1756-1774.

[22] R. Mercier, T. Schmitt, D. Veynante, B. Fiorina, Proc. Combust. Inst. 35 (2015) 1259-1267.

[23] T. Brauner, W. Jones, A. Marquis, Flow Turbul. Combust. 96 (2016) 965985

[24] J. Oijen, L. de Goey, Combust. Sci. Technol. 161 (2000) 113137.

[25] J. Oijen, R. Bastiaans, L. de Goey, Proc. Combust. Inst. 31 (2007) 1377-1384.

[26] C. B. da Silva, R. R. Taveira, Phys. Fluids 22 (2010) 121702.

[27] M. Holzner, M. van Reeuwijk, Journal of Turbulence 18 (2017) 260-270.

[28] T. Echekki, J. H. Chen, Combustion and Flame 106 (1996) 184202.

[29] T. Echekki, J. H. Chen, Combust. Flame 118 (1999) 308-311.

[30] T. Watanabe, Y. Sakai, K. Nagata, Y. Ito, T. Hayase, Physics of Fluids 26 (2014) 105103.

[31] M. Holzner, B. Lüthi, Physical review letters 106 (2011) 134503.

[32] C. Dopazo, L. Cifuentes, J. Martin, C. Jimenez, Combust. Flame 162 (2015) 1729-1736.

[33] C. Dopazo, L. Cifuentes, Combust. Sci. Technol. 188 (2016) 1376-1397.

[34] C. Dopazo, L. Cifuentes, N. Chakraborty, Phys. Fluids 29 (2017) 045106.

[35] C. Dopazo, J. Martin, J. Hierro, Phys. Rev. E. 76 (2007) 056316.

[36] L. Cifuentes, C. Dopazo, J. Martin, C. Jimenez, Phys. Fluids 26 (2014) 065108

[37] L. Cifuentes, C. Dopazo, J. Martin, P. Domingo, L. Vervisch, Proc. Combust. Inst. 35 (2015) 1295-1303.

[38] C. Dopazo, L. Cifuentes, J. Hierro, J. Martin, Flow Turbul. Combust. 96 (2016) 547-571.

[39] N. Chakraborty, I. Konstantinou, A. Lipatnikov, Phys. Fluids 28 (2016) 015109 


\section{Inner Interface}

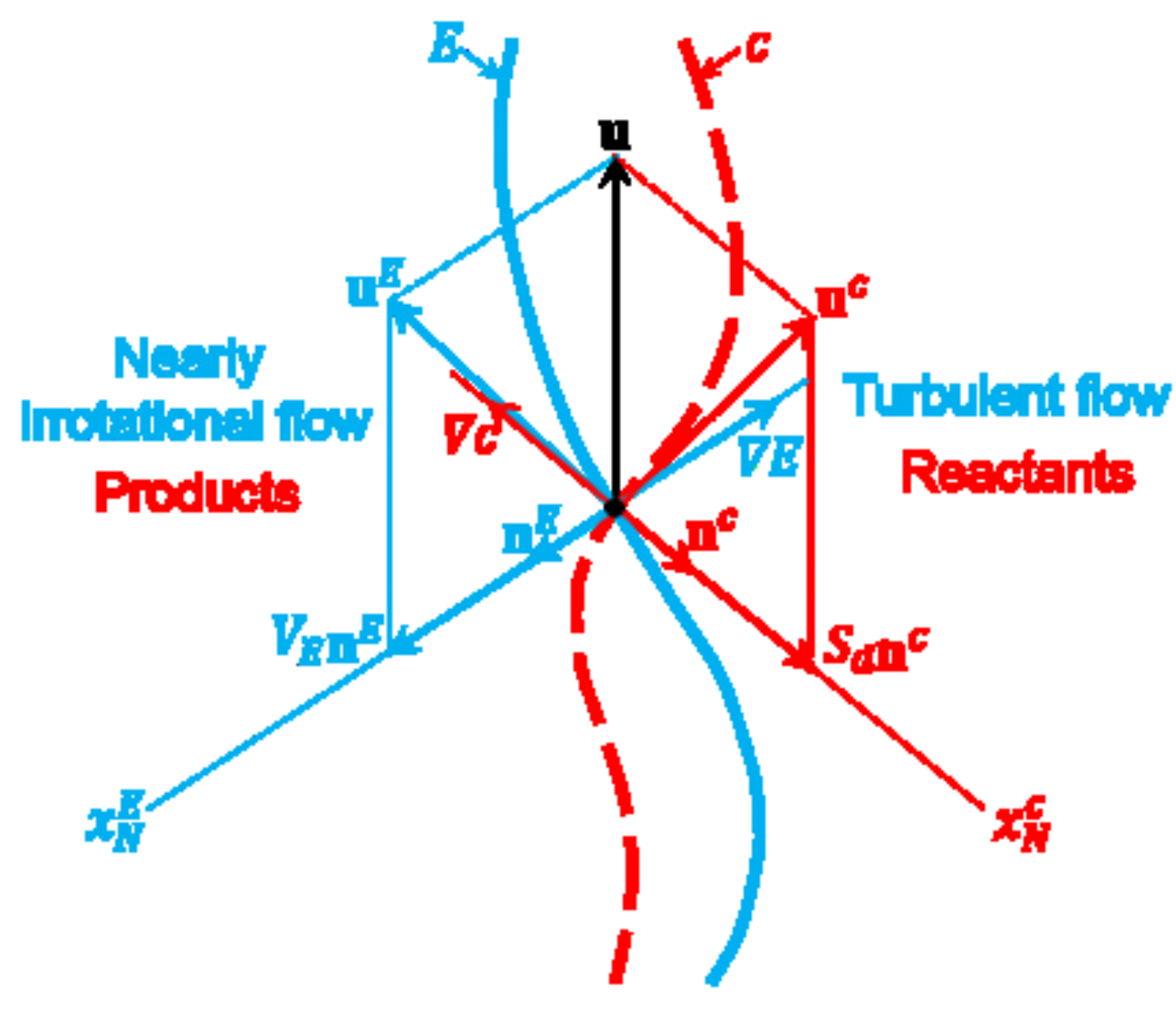

\section{Outer Interface}

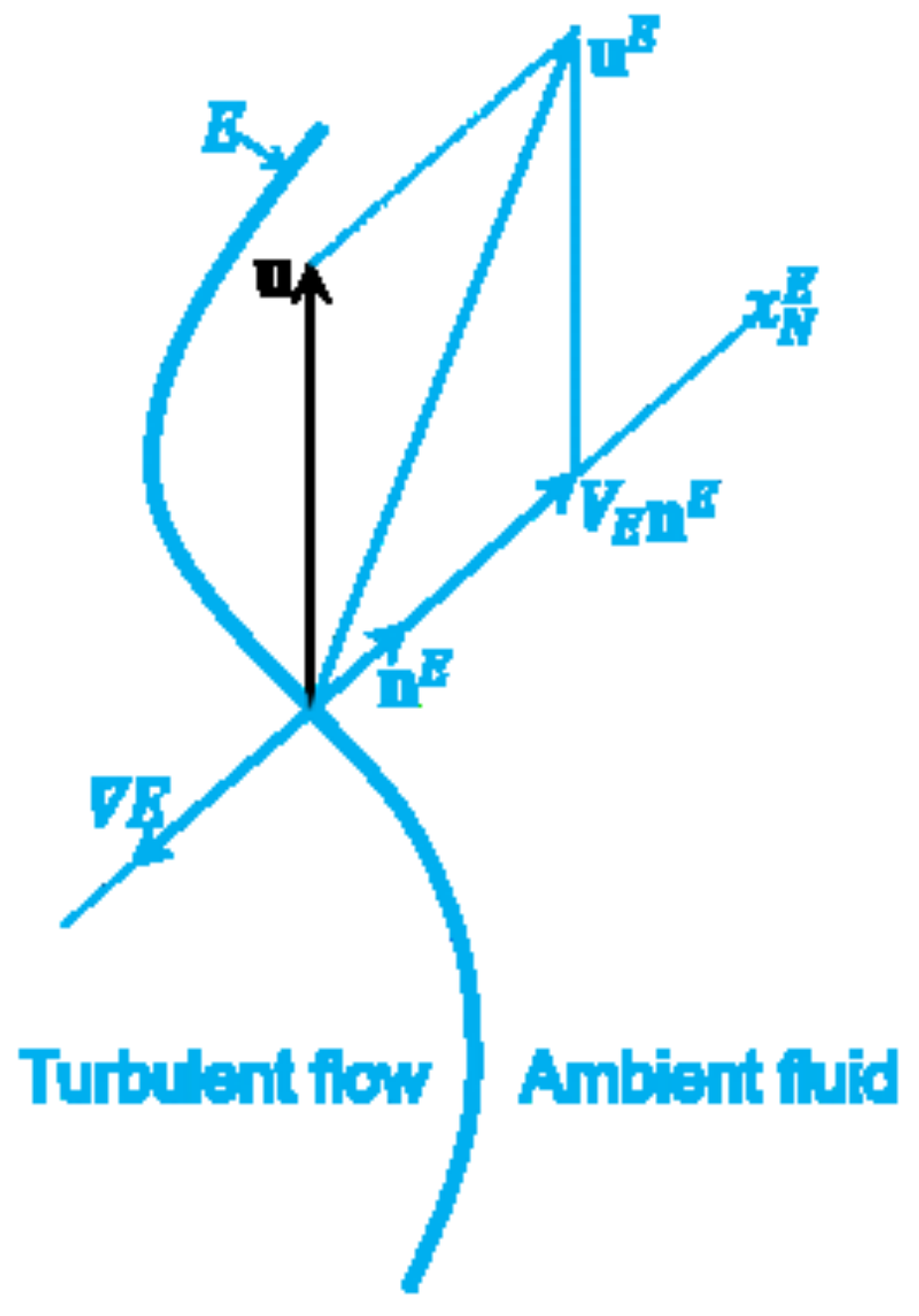




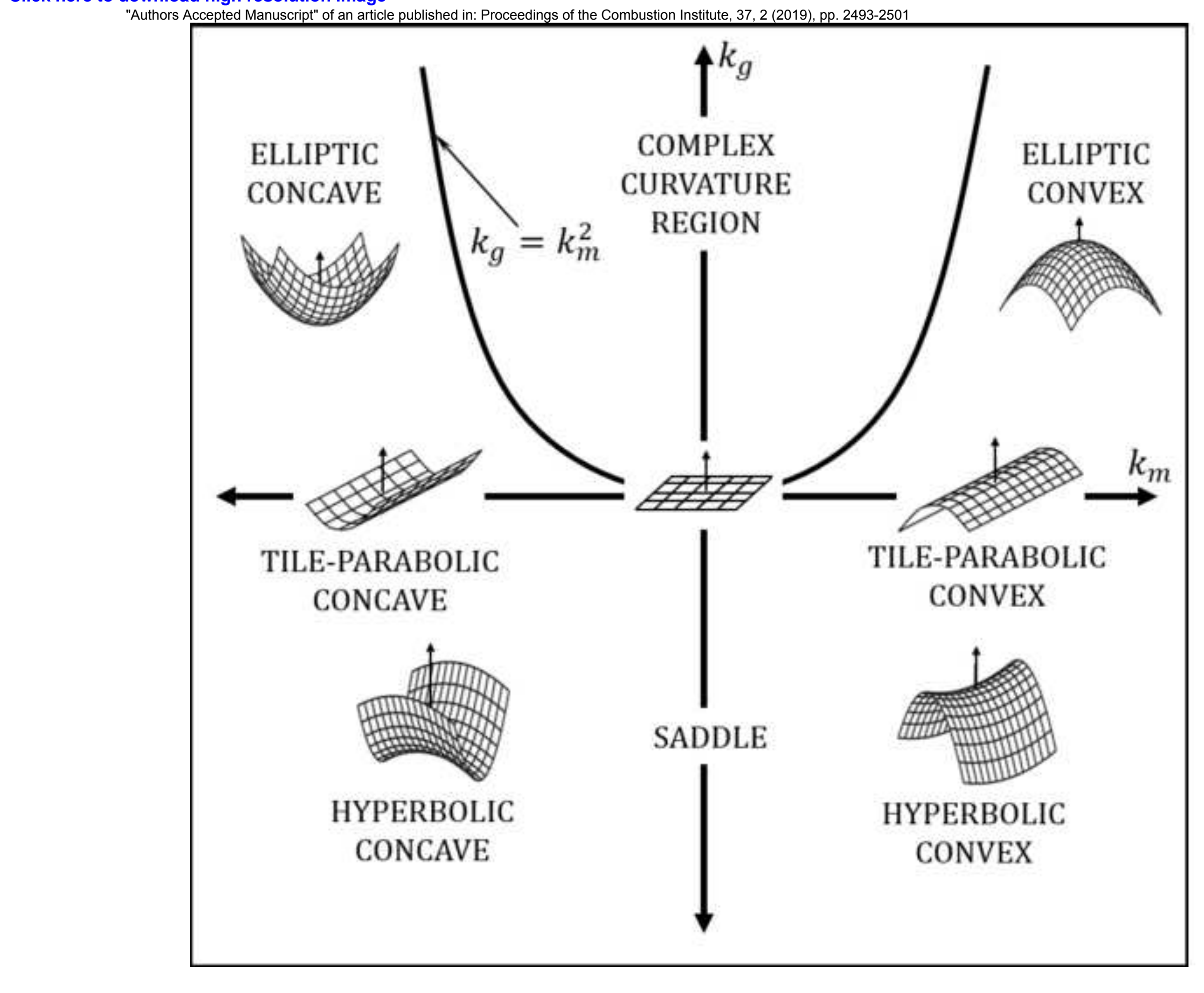

. 
Click here to download high resolution image

"Authors Accepted Manuscript" of an article published in: Proceedings of the Combustion Institute, 37, 2 (2019), pp. 2493-2501

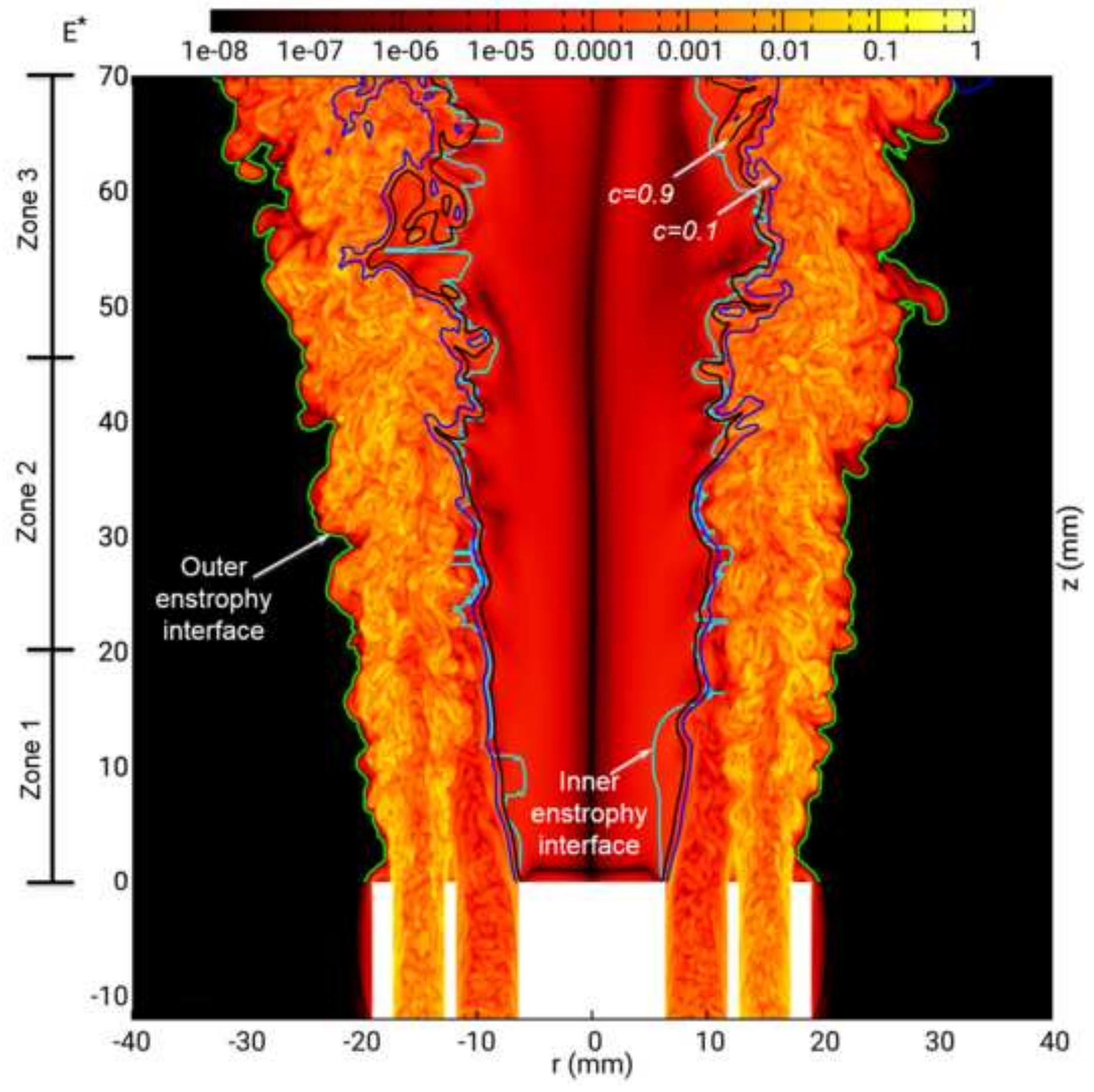

$\frac{E}{E}$ 


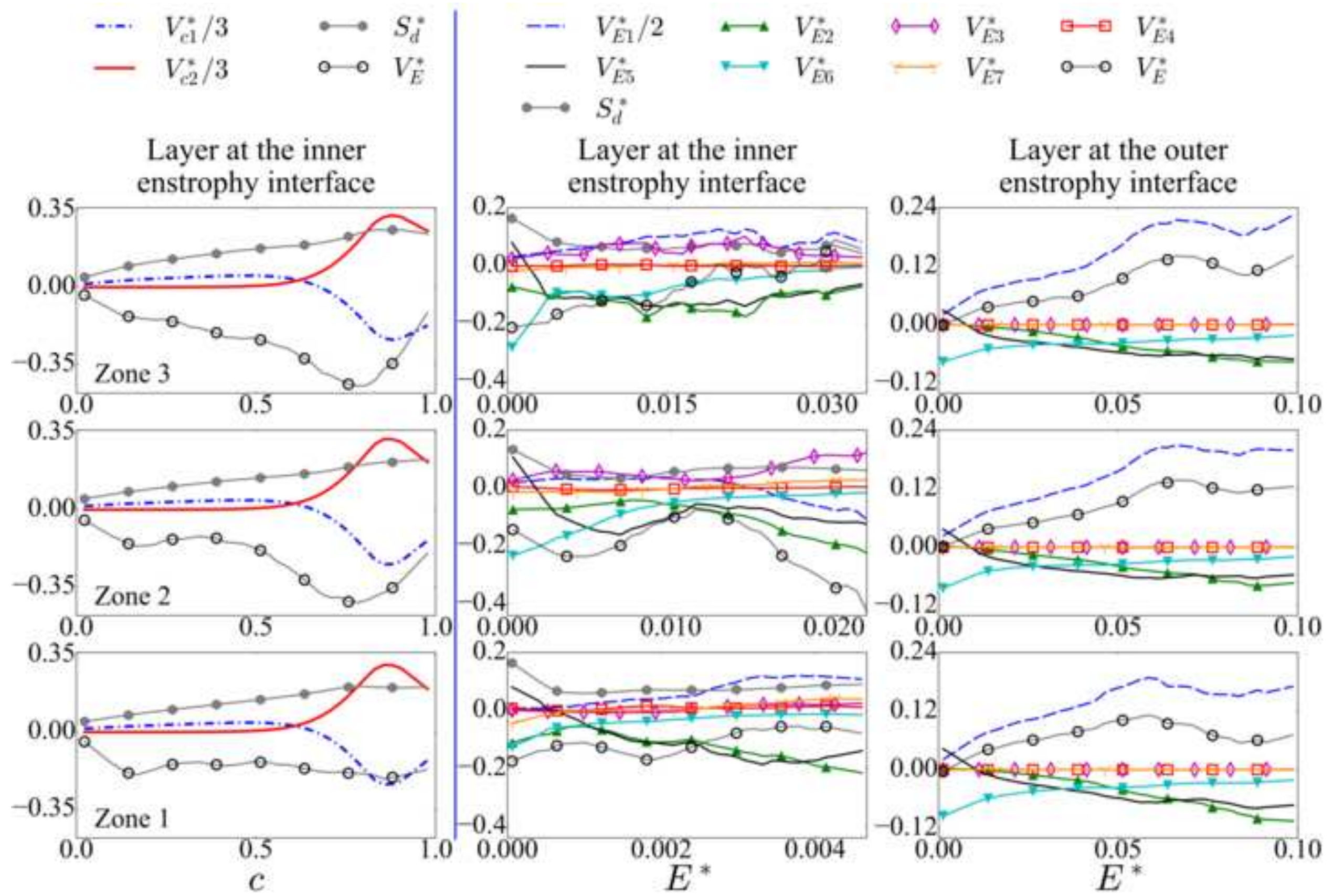

$$
\text { -.. } \quad V_{\text {el }}^{*} / 3
$$

Layer at the inner

0.12

$0.000-0.000-0000-000$ 


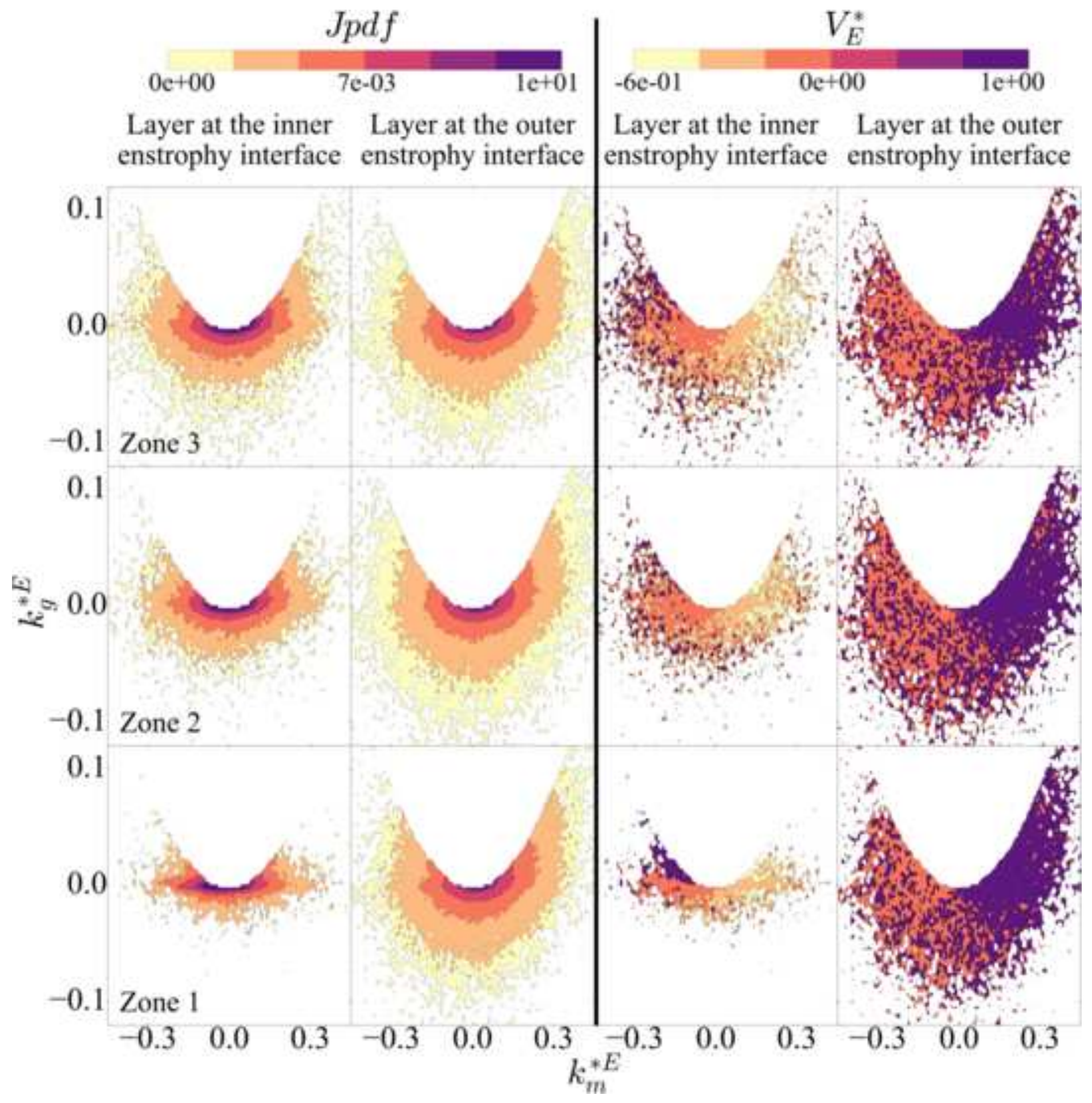




\title{
Local entrainment velocity in a premixed turbulent annular jet flame
}

\author{
Cifuentes, Luis; Kempf, Andreas; Dopazo, Cesar
}

This text is provided by DuEPublico, the central repository of the University Duisburg-Essen.

This version of the e-publication may differ from a potential published print or online version.

DOI: https://doi.org/10.1016/j.proci.2018.07.031

URN: urn:nbn:de:hbz:464-20190801-130142-7

Link: https://duepublico.uni-duisburg-essen.de:443/servlets/DocumentServlet?id=47990

License:

(cc) $(\mathrm{i}) \mathrm{BY}$

4.0 International license.

Source: This is the "Authors Accepted Manuscript" of an article published in:

Proceedings of the Combustion Institute, Volume 37, Issue 2, 2019, Pages 2493-2501; Available online 25 July 2018.

The final version may be found at: https://doi.org/10.1016/j.proci.2018.07.031 